\title{
Study and Application of Polymer Film-Forming Shielding Plugging Drilling Fluid System
}

\author{
Yuxue Sun, Fei Teng, and Jingyuan Zhao \\ Northeast Petroleum University, Daqing, Heilongjiang 163318, China \\ Correspondence should be addressed to Fei Teng; 476122791@qq.com
}

Received 11 October 2013; Revised 3 January 2014; Accepted 3 January 2014; Published 13 February 2014

Academic Editor: Alireza Bahadori

Copyright (C) 2014 Yuxue Sun et al. This is an open access article distributed under the Creative Commons Attribution License, which permits unrestricted use, distribution, and reproduction in any medium, provided the original work is properly cited.

\begin{abstract}
To solve the low cementing quality of acoustic logging and some distensible well diameters in high permeable layer of Daqing Lamadian oilfield, we have carried out the study and field application of polymer film-forming shielding plugging drilling fluid system. By adding this polymer film-forming shielding plugging agent to macromolecule emulsion drilling fluid, the shielding and plugging effect will be improved. Combined with the experiment and analysis, we optimize the adding amount of this agent to improve the performance of drilling fluid system. From the application effect, the cementing quality of acoustic logging has been improved obviously. This system has been applied in 20 wells and there are 15 high quality wells. The merit factor of well cementing reaches $75 \%$
\end{abstract}

\section{Introduction}

1.1. General Situation of Daqing Lamadian Oilfield. Daqing Lamadian oilfield is the central depression area of Songliao basin. It is an anticline sandstone reservoir in the extreme north of Daqing Changyuan area. This oilfield was developed in 1973. Through 30 years of development adjustment, it has experienced the adjustments of layer series, secondary infilling, and polymer flooding in main layer and so on. At present production has entered high water cut stage, and composite water cut has reached $94 \%$. Due to many years of injection and production, the formation characteristics have certain changes. From the site statistics, we found that the permeability had a tendency to increase with the development of Lamadian oilfield. At the same time, the indoor test using formation water to flood the natural core of Lamadian area for long term showed that the clay volume decreased after long-term waterflooding. But both permeability and porosity increased. In addition, Lamadian oilfield often used produced water to recirculate, so the formation water became calcium bicarbonate water. The total mineralization was as high as $7150 \mathrm{mg} / \mathrm{L}$. Therefore, the most prominent features of Lamadian oilfield are high porosity, high permeability, high water cut, and high salinity. Due to long-term waterflood development of Putaohua oil layer, the layer was serious water flush. Cementing of sandstone reservoir became loose, and rock strength decreased obviously. Conventional drilling fluid could not form a compact mud cake. It made the slurry fluid loss become large and seriously affected cementing quality. In order to solve the above problems, the zero permeability and low permeability plugging drilling fluid technology has been carried out in Daqing Lamadian oilfield. The field test applied in tertiary infilling adjustment well drilling has obtained good effect. For better improving cementing quality of Daqing Lamadian oilfield, our laboratory carried out the study and field application of polymer film-forming shielding plugging drilling fluid technology [1-6].

1.2. Outline of This Paper. Based on the current situation of Lamadian oilfield, we study the function mechanism of this polymer film-forming shielding plugging drilling fluid. In Section 3, we first use the new sand bed experimental method to test the plugging ability. According to the experimental results we optimize the adding amount of film-forming agent and analyze this new drilling fluid system. Finally we study the performance of this optimized drilling fluid system and extend it to the field application. In this paper, the novel point lies in the optimization of polymer film-forming shielding plugging drilling fluid system. This system, compatible with Daqing Lamadian strata, can improve the cementing quality of high permeable layer in Daqing Lamadian area obviously. 


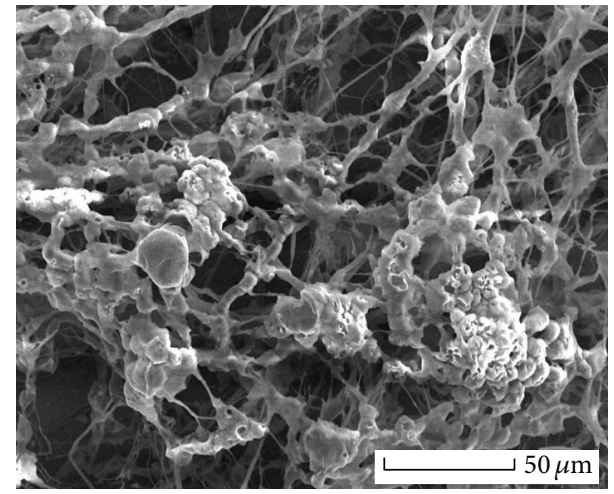

FIGURE 1: Water immersion of natural modified macromolecule compound for 1 hour.

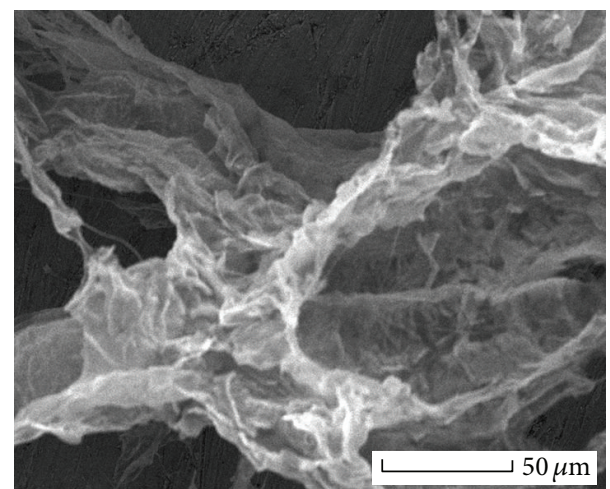

FIGURE 2: Water immersion of natural modified macromolecule compound for 24 hours.

\section{Mechanism Study}

This technology mainly uses special polymer addictive to concentrate into micelle in high permeable sandstone pore or microcrack port. We rely on the interface suction and deformability of polymer micelle or colloidal particle, and cooperate with inert solid particles or fibers. Under gradual swelling effect of the polymer particles, we form a dense ultralow permeability sealing film on the wellbore surface [7-10].

2.1. Swelling Mechanism of Special Polymer Additive. This polymer addictive is a kind of natural modified macromolecular compound. The colloid contains lots of hydration groups. It can absorb water to form micelle. The micelle, macromolecule group, can swell by absorbing water, but it cannot dissolve completely. Because of the swelling characteristic of natural macromolecule compound, we can add hydrophobic molecules, which can prevent water molecules entering into micelle; thus water intake rate of natural macromolecule polymer will be delayed to achieve the goal of gradual water absorption and slow swelling. Due to the strong effect of cross-linked network, the polymer only swells not dissolves. Figures 1 and 2 are the water absorption swelling changes of natural macromolecule compound in 24 hours. From the two figures, we can see that both the swelling micelle density and grain diameter of micelle became large 24 hours later [11-14].

2.2. Plugging Mechanism. Using the characteristics of natural modified polymer and filling effect of inert particles and

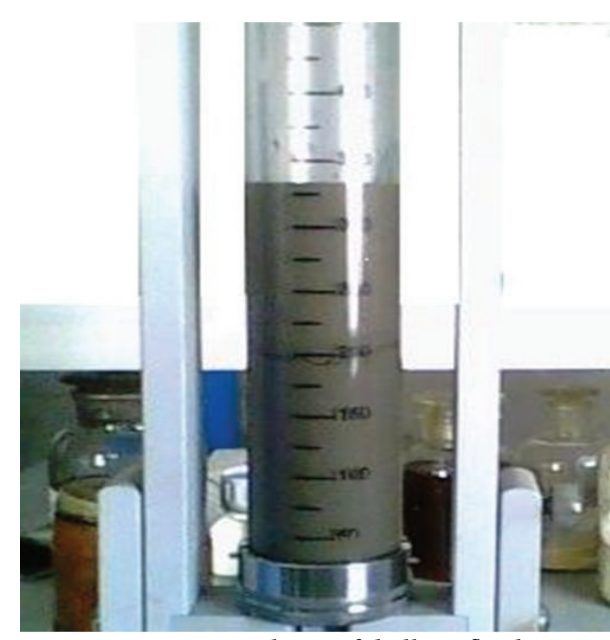

FIGURE 3: Leakage of drilling fluid.

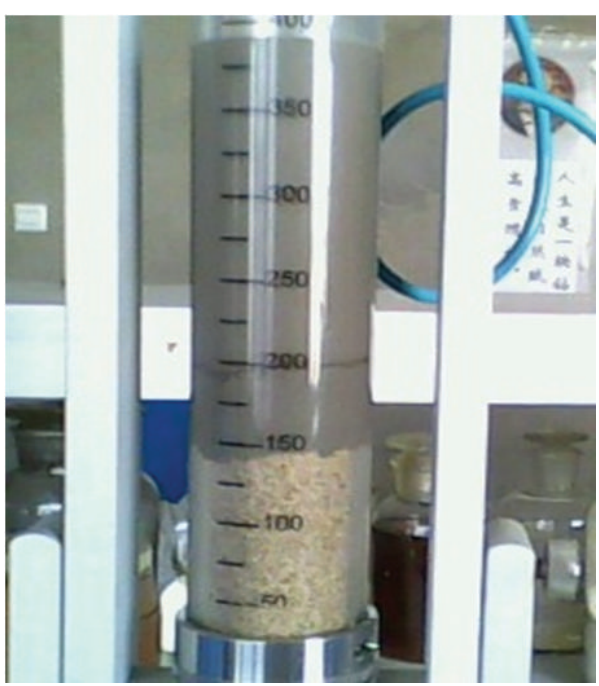

FIGURE 4: Plugging of drilling fluid.

fibers will make the whole system have a good plugging effect. First, we match the inert materials reasonably by the optimization accumulation theory. Through the particle accumulation and bridge connection of fiber, we form a compacted mud cake. Then the entire sealing interface volume will increase gradually by water absorbing swelling effect of natural macromolecule. When the volume swells to a certain extent, the antipressure ability can be strengthened and particles in the sealing interface cannot divorce from plugged zone to the recesses of pore and fracture [15-17]. At the same time it can prevent drilling fluid and filtrate seeping into the formation. It makes near-zero filtration drilling come true and protects reservoir effectively.

\section{Materials and Methods}

3.1. Optimizing the Adding Amount of Polymer Film-Forming Shielding Plugging Agent. Using the new sand bed experimental method, we carried out the experiment to test the plugging ability and adding amount of this addictive. Figures 3 and 4 are the visual images of sand bed experiment. Experimental data is shown in Table 1. 
TABLE 1: Sand bed leakage experiment.

\begin{tabular}{lccc}
\hline Formula & $\begin{array}{c}\rho \\
\left(\mathrm{g} / \mathrm{cm}^{3}\right)\end{array}$ & $\begin{array}{c}\text { FL } \\
(\mathrm{mL})\end{array}$ & $\begin{array}{c}\text { Experiment condition } \\
(0.7 \mathrm{MPa}, 30 \mathrm{~min}, 20 \sim 40 \mathrm{mesh} \text { quartz sand, room temperature })\end{array}$ \\
\hline $\begin{array}{l}\text { Emulsion slurry } \\
\text { system } \\
\text { Slurry + 1\% MFDJ }\end{array}$ & 1.49 & 4 & $\begin{array}{c}\text { Instantaneous loss } \\
\text { Instantaneously soak to } 6 \mathrm{~cm} \text { and } 10 \text { minutes later soak to } 8.5 \mathrm{~cm} \text {. There is no } \\
\text { increase. Forcing to } 1.2 \mathrm{MPa} \text {, there are also no changes. }\end{array}$ \\
$\begin{array}{l}\text { Slurry + 2\% MFDJ } \\
\text { Slurry + 3\% MFDJ }\end{array}$ & 1.49 & 3.6 & $\begin{array}{c}\text { Instantaneously soak to } 2.8 \mathrm{~cm} \text { and } 10 \mathrm{minutes} \text { later soak to } 3.5 \mathrm{~cm} \text {. There is no } \\
\text { increase. Forcing to } 1.2 \mathrm{MPa} \text {, there are also no changes. }\end{array}$ \\
Slurry + 4\% MFDJ & 1.50 & 2.8 & $\begin{array}{r}\text { Instantaneously soak to } 2 \mathrm{~cm} \text { and } 10 \mathrm{minutes} \mathrm{later} \mathrm{soak} \mathrm{to} 2.5 \mathrm{~cm} \text {. There is no } \\
\text { increase. Forcing to } 1.2 \mathrm{MPa} \text {, there are also no changes. }\end{array}$ \\
\hline
\end{tabular}

MFDJ is a kind of polymer film-forming shielding plugging addictive.

TABLE 2: Compatible evaluation indoor experiment of various adding amounts.

\begin{tabular}{|c|c|c|c|c|c|c|c|}
\hline Formula & $\begin{array}{c}\rho \\
\left(\mathrm{g} / \mathrm{cm}^{3}\right)\end{array}$ & $\begin{array}{r}\mathrm{AV} \\
(\mathrm{s})\end{array}$ & $\begin{array}{c}\mathrm{PV} \\
(\mathrm{mPa} \cdot \mathrm{s})\end{array}$ & $\tau_{0} / \mu_{p}$ & $\begin{array}{c}\text { Gel10" }^{\prime \prime} / \mathrm{Gell}^{\prime} \\
(\mathrm{Pa})\end{array}$ & $\begin{array}{l}\mathrm{FL} \\
(\mathrm{mL})\end{array}$ & $\begin{array}{l}\text { Mud } \\
\text { cake } \\
(\mathrm{mm})\end{array}$ \\
\hline Emulsion slurry system & 1.49 & 36 & 25.5 & 0.36 & $1 / 2.5$ & 4.4 & 1.0 \\
\hline Slurry + 1\% MFDJ & 1.49 & 37 & 26.5 & 0.4 & $1 / 3$ & 4.2 & 1.0 \\
\hline Slurry + 2\% MFDJ & 1.49 & 40 & 29 & 0.45 & $1.5 / 3.5$ & 3.6 & 1.0 \\
\hline Slurry + 3\% MFDJ & 1.50 & 44 & 32.5 & 0.51 & $2 / 4$ & 3 & 1.0 \\
\hline Slurry + 4\% MFDJ & 1.51 & 53 & 38 & 0.58 & $2.5 / 5$ & 2.6 & 1.2 \\
\hline
\end{tabular}

TABLE 3: Measured drilling fluid performance of La 5-PS1922.

\begin{tabular}{|c|c|c|c|c|c|c|c|c|c|}
\hline $\begin{array}{l}\text { Depth } \\
(\mathrm{m})\end{array}$ & $\begin{array}{c}\rho \\
\left(\mathrm{g} / \mathrm{cm}^{3}\right)\end{array}$ & $\begin{array}{r}\mathrm{AV} \\
(\mathrm{s})\end{array}$ & $\begin{array}{c}\mathrm{FL} \\
(\mathrm{mL})\end{array}$ & $\begin{array}{l}\text { Mud cake } \\
(\mathrm{mm})\end{array}$ & $\begin{array}{l}\mathrm{YP} \\
(\mathrm{Pa})\end{array}$ & $\begin{array}{c}\mathrm{PV} \\
(\mathrm{mPa} \cdot \mathrm{s})\end{array}$ & $\tau_{0} / \mu_{p}$ & $\begin{array}{c}\text { Gel10 }^{\prime \prime} / \mathrm{Gell}^{\prime} \\
(\mathrm{Pa})\end{array}$ & $\begin{array}{c}R \\
\left(\Omega \cdot \mathrm{M} / 18^{\circ} \mathrm{C}\right) \\
\end{array}$ \\
\hline 850 & 1.35 & 52 & 4.8 & 1.0 & 9.5 & 21 & 0.45 & $2 / 4$ & 4.10 \\
\hline 910 & 1.48 & 68 & 3.4 & 1.0 & 19 & 33 & 0.58 & $5 / 7.5$ & 3.55 \\
\hline 945 & 1.48 & 62 & 3.8 & 1.0 & 15 & 28 & 0.54 & $4.5 / 6.5$ & 3.57 \\
\hline 1035 & 1.48 & 60 & 3.6 & 1.0 & 14.5 & 27 & 0.54 & $4 / 6$ & 3.68 \\
\hline 1084 & 1.48 & 57 & 3.8 & 1.0 & 13 & 27 & 0.48 & $3 / 7$ & 3.75 \\
\hline Electric log & 1.48 & 55 & 3.6 & 1.0 & 10 & 25 & 0.40 & $3 / 5$ & 3.55 \\
\hline Cementation & 1.48 & 55 & 3.6 & 1.0 & 10 & 25 & 0.40 & $3 / 5$ & \\
\hline
\end{tabular}

3.2. Conventional Performance Experiment of Polymer FilmForming Shielding Plugging Additive. We conduct the conventional performance comparison experiment by adding different amount of this addictive into the in situ macromolecule emulsion drilling fluid system. Data is shown in Table 2 .

\section{Results and Discussion}

4.1. Optimizing the Adding Amount of Polymer Film-Forming Shielding Plugging Agent. From Table 1, sand bed leakage experiment data shows that MFDJ has good film-forming plugging ability. It can well prevent sandstone reservoir from seepage and improve the bearing capacity of borehole wall. Adding $1 \%$ of this addictive into the emulsion polymer drilling fluid can realize the zero filtration. The immersion depth into sand bed is $8.5 \mathrm{~cm}$. When adding $2 \%$, the depth will become $3.5 \mathrm{~cm}$. Adding 3\% and $4 \%$, the depth is $2.5 \mathrm{~cm}$ and $2 \mathrm{~cm}$, respectively. The adding amount of this addictive can be determined in the range of $2 \%$ to $4 \%$.

4.2. Function Morphology and Microstate of Polymer FilmForming Shielding Plugging Additive. We observe the surface of sand bed after the experiment and of mud cake after medium voltage loss experiment (shown in Figure 5). From the phenomenon of sand bed plugging experiment, we can see that modified high molecular polymer and inert particle material are embedded into the sand bed surface pore. Filter cake of drilling fluid presents dense reticular structure. Thus it can achieve the effect of blocking and strengthening borehole wall [10].

4.3. Conventional Performance of Polymer Film-Forming Shielding Plugging Additive. From Table 2, the experimental 


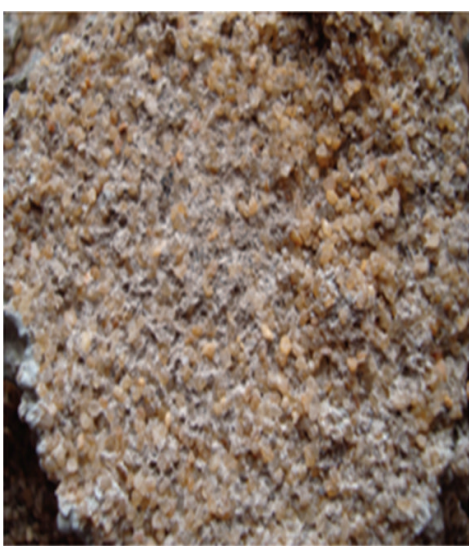

Surface state of sand bed after drilling fluid penetration

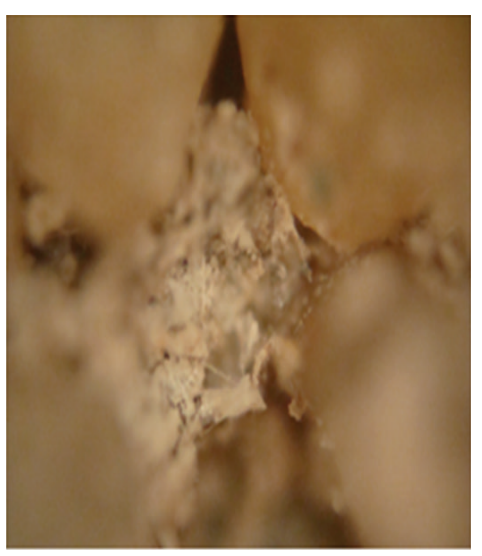

Drilling fluid microcrystalline state of embedding into sand bed pore

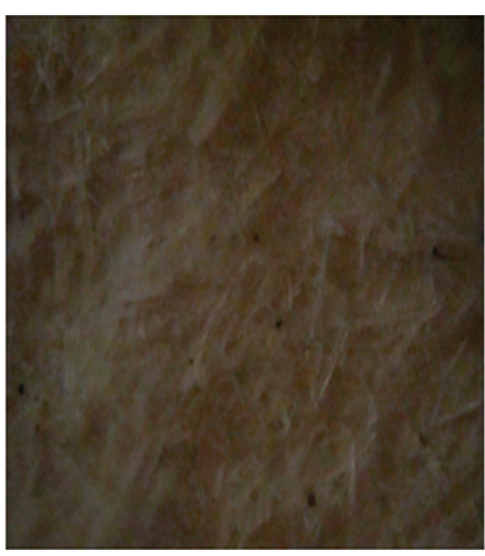

State of drilling fluid filter cake surface

FIGURE 5: Photos of sand bed surface after new sand bed experiment and mud cake surface of medium voltage loss experiment.

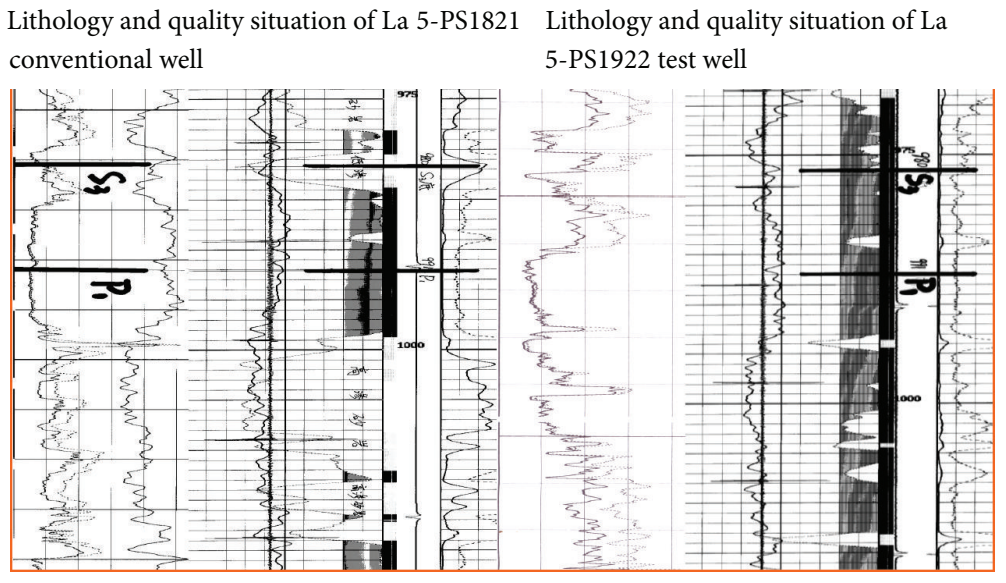

Figure 6: Contrast picture of cementing time-delay acoustic change detection.

data shows that when the slurry is normal formula of emulsion macromolecule drilling fluid system and the adding amount is less than $3 \%$, the effect on density, viscosity, yield point and plastic viscosity ratio, and mud cake thickness of drilling fluid is smaller. When the adding amount is $4 \%$, the parameter values of apparent viscosity, plastic viscosity, and shearing force increase obviously. The difficulty of protecting the drilling fluid rheological properties also increases correspondingly. According to the immersion depth into sand bed and rheological properties of drilling fluid, we optimize synthetically the adding amount of $2 \% \sim 3 \%$.

\subsection{Field Application}

4.4.1. Application Scheme. Based on indoor experimental data, we determine the amount of this additive to 3 tons per well. The applied interval is from weighting to finishing drilling. The specific addition method is to adjust drilling fluid properties after weighting and then to add this addictive 2 tons at a time. To maintain the effective adding amount of this addictive in the drilling fluid, after weighting, it needs to be supplied gradually in the drilling fluid. One ton addictive should be supplied before entering the P1 group.

4.4.2. Field Application. This drilling fluid technology has been applied into La 5-PS1922 well of 15556 drilling crew on September 6, 2010. During field test process, we adjust drilling fluid properties after weighting and we add 2 tons treated addictive at a time. During the adding process, the drilling fluid viscosity increased slightly. The drilling fluid viscosity returned to normal after adding some diluting addictive to maintain the viscosity. In the subsequent drilling process, 1 ton treatment addictive was supplied into drilling fluid successively. The electric log and cementing operation of this well was normal. The measured drilling fluid performance in the field is shown in Table 3.

4.4.3. Application Effect. Drilling fluid technology of polymer film-forming shielding plugging was applied to 20 wells of Lamadian oilfield. Site construction was smooth without any complicated engineering problems. Successful rate of electric $\log$ was $100 \%$. The merit factor of cementing time-delay 
acoustic change detection was $75 \%$, and qualification rate was $100 \%$. Figure 6 is the contrast picture of cementing time-delay acoustic change detection of high permeable layer in a La 5PS1922 test well.

From Figure 6, we can see that after applying this treatment addictive in the field, the cementation quality of high permeable layer S3 and P1 group in La 5-PS1922 is improved obviously compared with La 5-PS1821 well of conventional drilling fluid construction.

\section{Conclusions}

Indoor sand bed experiment shows that the polymer filmforming shielding plugging addictive can form a better compact and low permeable mud cake on the high permeable layer. The function mechanism in film-forming shielding plugging materials, which can effectively improve the low permeability property of drilling fluid, is the swelling effect of modified macromolecule polymer. In the optimized experiment, the best adding amount of polymer film-forming shielding plugging addictive in high permeable layer is $3 \%$ $5 \%$. And field application effect shows that this drilling fluid technology can improve the time-delay acoustic change cementing quality of Lamadian high permeable layer obviously.

\section{Conflict of Interests}

The authors declare that there is no conflict of interests regarding the publication of this paper.

\section{Acknowledgment}

This study is supported by Scientific Research Fund of Heilongjiang Provincial Education Department (no. 12521045).

\section{References}

[1] J. Sun, J. Zheng, D. Huang, and B. Wang, "Study and application of ultra-low permeable drilling fluid: lost circulation prevention and control," Drilling Fluid and Completion Fluid, vol. 22, no. 4, pp. 21-24, 2005.

[2] J. Sun, X. Lin, B. Zhang et al., "Abroad technology summary of ultra-low permeable drilling fluid," Drilling Fluid \& Completion Fluid, vol. 22, no. 1, pp. 57-59, 2005.

[3] J. Sun, Y. Su, P. Luo, and Y. Liu, "Mechanism research of ultralow permeable drilling fluid to improve formation bearing capacity," Drilling Fluid \& Completion Fluid, no. 5, pp. 1-3, 2005.

[4] B. Jing, W. Sui, and C. Pei, "Research and application of ultra-low permeable drilling fluid in H17 oilfield," Drilling \& Production Technology, no. 5, pp. 36-39, 2008.

[5] Y. Peng, Y. Liu, Y. Song, P. Qing, and Y. Lu, "Application of ultralow invasion fluid technology in Xiaolongwan block," Drilling Fluid \& Completion Fluid, vol. 22, no. 5, pp. 31-34, 2005.

[6] F. Zhou, S. Tang, Y. Wang, and T. Huang, "The application of ultra-low filter loss drilling fluid in well Hua 3-8," Drilling Fluid \& Completion Fluid, vol. 24, no. 6, pp. 29-31, 2007.

[7] J. Yan, Drilling Fluid Technology, 1st version in May, 2001, Petroleum University Press, Dongying, China, 2003.
[8] P. Reid and H. Santos, "Novel drilling, completion and workover fluids for depleted zones: avoiding losses, formation damage and stuck pipe," in Proceedings of the SPE IADC, October 2003.

[9] H. Santos, M. B. Villas-Boas, R. F. T. Lomba, C. H. M. Sa, S. F. Oliveira, and J. F. Costa, "API filtrate and drilling fluid invasion: is there any correlation," in Proceedings of the Latin American and Caribbean Petroleum Engineering Conference, Caracas, Venezuela, April 1999.

[10] H. Santos and R. Perez, "What have we been wrong in wellbore stability?" in Proceedings of the SPE Latin American and Caribbean Petroleum Engineering Conference, Buenos Aires, Argentina, March 2001.

[11] J. Xie, Z. Wang, B. Zhang, and X. Wang, "New drilling fluid system and plugging technology in Zhongyuan oilfield," Advance in Fine Petrochemical Complex, no. 1, pp. 1-5, 2010.

[12] M. Arabloo, M. P. Shahri, and M. Zamani, "Characterization of colloidal gas aphron drilling fluids produced from a new plant based surfactant," Journal of Dispersion Science and Technology, vol. 34, pp. 669-678, 2013.

[13] S. Luo, Y. Li, Y. Meng, and L. Zhang, "A new drilling fluid for formation damage control used in underbalanced drilling," in Proceedings of the IADC/SPE Drilling Conference, New Orleans, La, USA, February 2000.

[14] S. Zhang, G. Jiang, H. Guo, X. Tang, and H. Jin, "Study of new film-forming agent in drilling fluid system and application in Chenghai oilfield," Petroleum Drilling Techniques, vol. 41, no. 2, pp. 44-47, 2013.

[15] Y. Chun, S. Jinsheng, W. Pingquan et al., "Development of CMJ1: a high temperature film-forming fluid loss additive and the properties," Petroleum Drilling Techniques, vol. 32, no. 2, pp. 3032, 2004.

[16] B. Xiaodong and P. Xiaolin, "Evolution of membrane forming technology of water-based mud," Natural Gas Industry, vol. 26, no. 8, pp. 75-77, 2006.

[17] L. Haitao, Z. Xiutai, L. Qiulian et al., "Laboratory study on drilling fluid of mosaic agent-membrane agent synergistic interaction for reservoir protection," Petroleum Drilling Techniques, vol. 40, no. 4, pp. 65-71, 2012. 

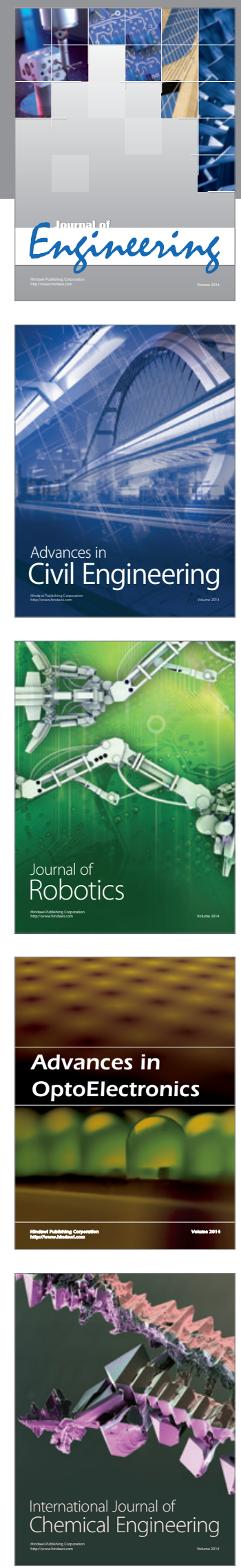

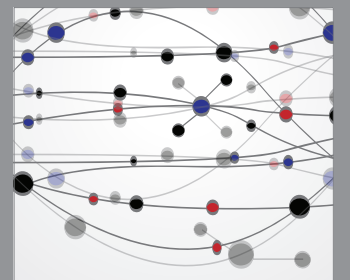

The Scientific World Journal
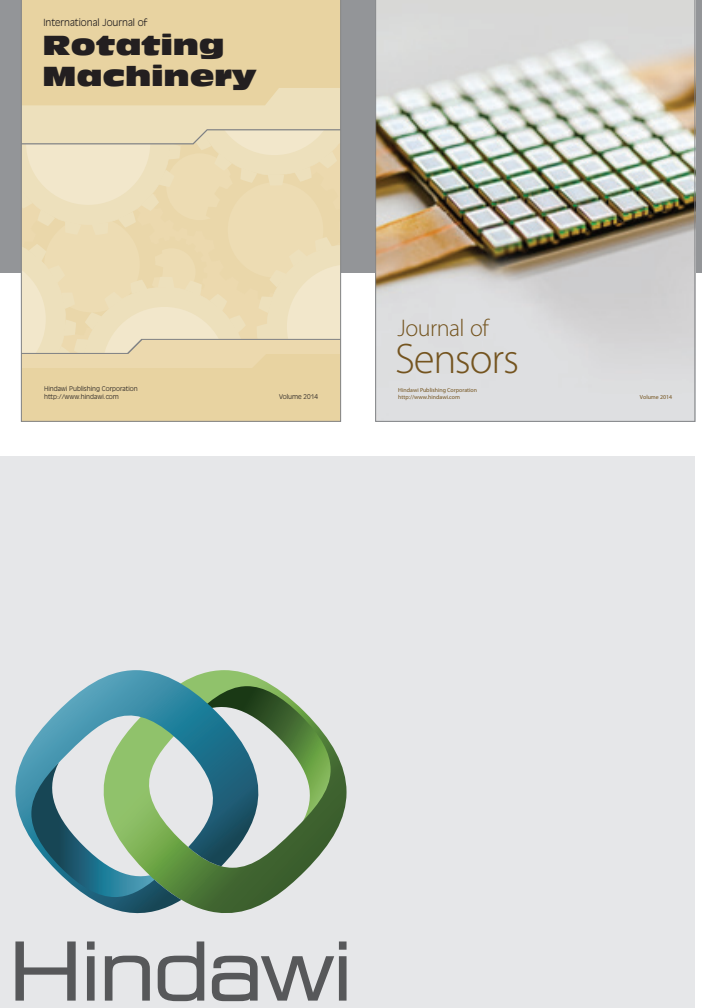

Submit your manuscripts at http://www.hindawi.com
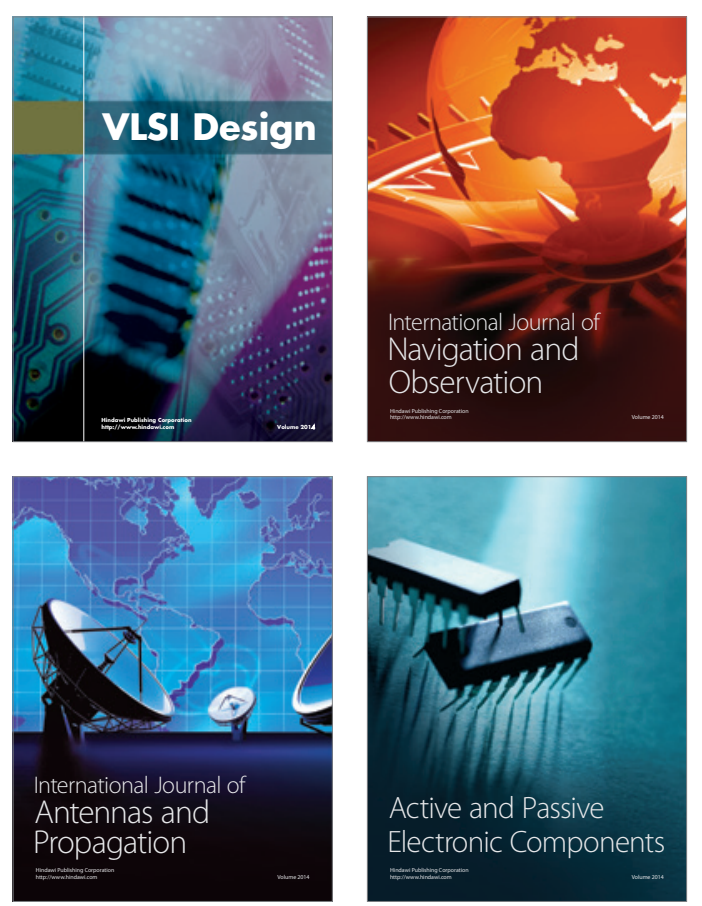
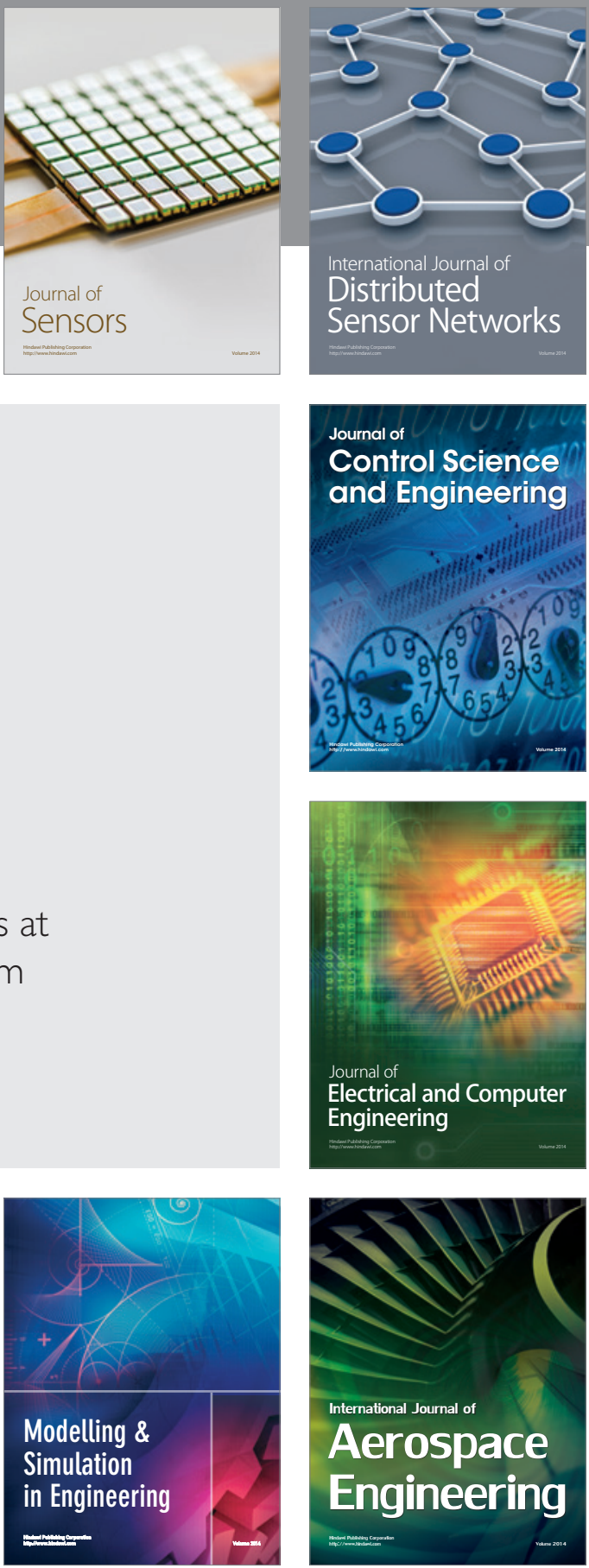

Journal of

Control Science

and Engineering
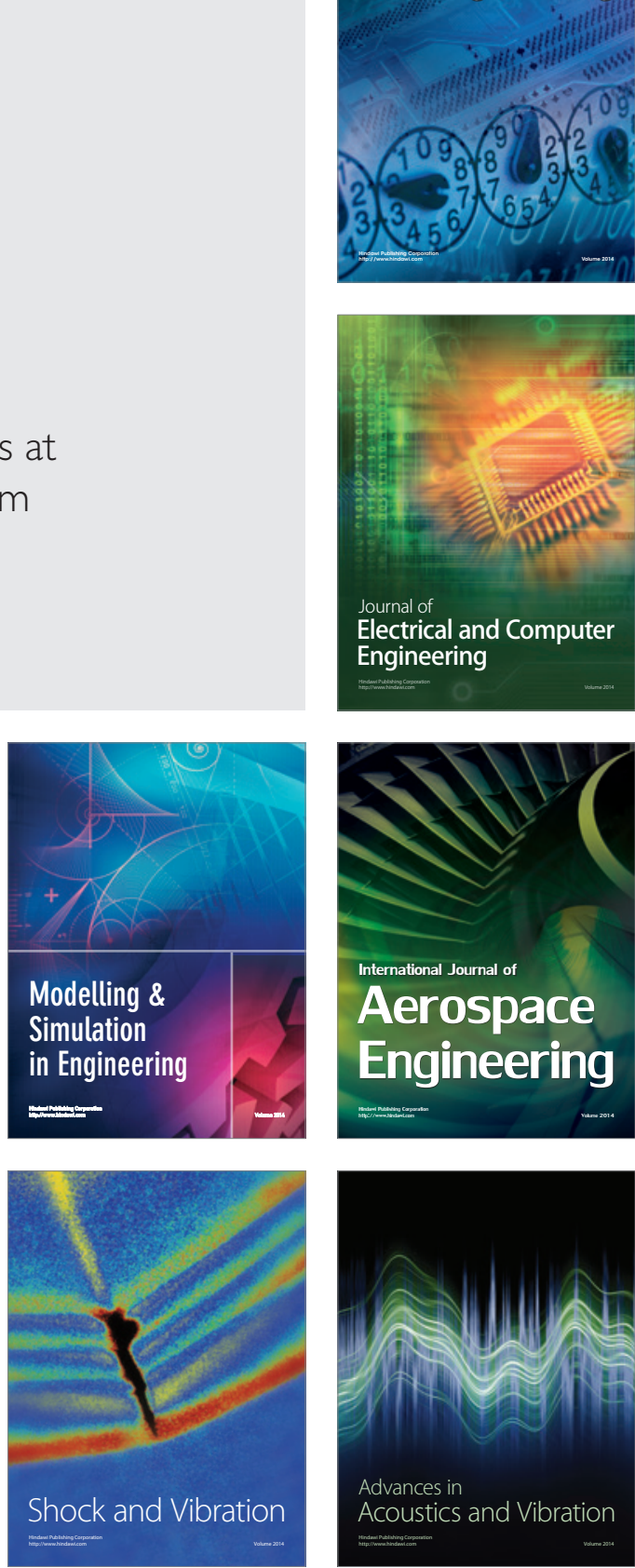